\title{
Nephroblastoma: Radiological and Pathological Diagnosis of a Case with Liver Metastases
}

\author{
Kouamé Ngoran1*, Doukouré Brahima², Manewa Fotso Sorelle1, Gaimou Blé P1, \\ Yessoufou Bakary Nafissath ${ }^{3}$, N'Goan-Domoua Anne-Marie ${ }^{1}$ \\ ${ }^{1}$ Department of Radiology at the University Hospital of Yopougon, Abidjan, Ivory Coast \\ ${ }^{2}$ Department of Pathology at the University Hospital of Cocody, Abidjan, Ivory Coast \\ ${ }^{3}$ Department of Radiology at the University Hospital Sylvanus Olympio, Lome, Togo \\ Email: "kngoran@yahoo.fr
}

Received 22 November 2015; accepted 12 March 2016; published 15 March 2016

Copyright (C) 2016 by authors and Scientific Research Publishing Inc.

This work is licensed under the Creative Commons Attribution International License (CC BY). http://creativecommons.org/licenses/by/4.0/

(c) (i) Open Access

\section{Abstract}

Nephroblastoma is one of the most common causes of abdominal-pelvic mass in children. It still raises a diagnostic problem because of the wide variety of causes of abdominal-pelvic mass but also because of the delays in consultation in sub-Saharan black Africa and consequently the discovery of the mass at a very late and sometimes metastatic stage. Yet nephroblastoma is a very chemo-sensitive malignancy requiring diagnosis at an early stage; a procedure in which medical imaging is essential. We report the case of a large abdominal-pelvic mass in a three-year-old girl in whom the abdominal-pelvic CT allowed to diagnose nephroblastoma with liver metastases. Our objective is to demonstrate the contribution of computed tomography in the diagnosis of large abdominalpelvic mass of the child and discuss other causes of abdominal-pelvic mass of the child.

\section{Keywords}

Wilms Tumor, Abdominal-Pelvic CT, Abdominal-Pelvic Mass, Nephroblastoma, Child

\section{Introduction}

Nephroblastoma or Wilms tumor, a common cause of abdominal mass [1] [2], is the most common kidney malignancy of the child [3]. This is a particular malignant tumor because of its favorable outcome on treatment [4]. Hence it needs to diagnose it at an early stage [3]. Medical imaging plays an important role in the positive diagnosis, staging, and monitoring of treatment [5]. Given the pediatric field, ultrasound and MRI are ideally the

\footnotetext{
*Corresponding author.
} 
dedicated means of imaging. But in practice, ultrasound is often exceeded given the volume of the mass; the echo guided puncture for histological diagnosis is contraindicated and MRI is not always available. The CT although very radiant remains essential in this approach. We report the case of a large abdominal-pelvic mass that occurred in a three-year-old girl in whom CT enabled the diagnosis of Wilms tumor with liver metastases. Our aim is to describe nephroblastoma semiology at the CT scan and discuss other causes of abdominal-pelvic mass of the child.

\section{Observation}

KMC is a 3-year-old girl. She was admitted to the pediatric department for afebrile abdominal bulging. On clinical examination she presented a discrete alteration of the general condition, a minor pallor of the conjunctiva and a large abdominal pelvic mass lateralized to the left. There was no lumbar contact. An ultrasound was required and brought out a large abdominal pelvic tumor of heterogeneous tissue structure with no visualization of the left kidney and a significant repression of the spleen, liver and right kidney. The ovaries and uterus were not visualized. An abdominal pelvic CT was requested to clarify the diagnosis. The apparatus used was a multi-cut scanner TOSHIBA. It was performed before and after injection of iodinated contrast medium according to the following protocol: an abdominal-pelvic volume acquisition in spontaneous contrast. 3 acquisitions after injection of iodinated contrast medium (at a dose of $2 \mathrm{ml} / \mathrm{kg}$ ) at the arterial times (30 seconds), portal (70 seconds) and late ( 3 minutes). The mass was of tissue density ( $55 \mathrm{UH}$ ) and moderately heightened after injection of iodinated contrast medium. It measured $132 \mathrm{~mm}$ in diameter and was very well limited. Its scan was heterogeneous without image of internal calcifications (Figure 1). The left kidney was visualized after injection of iodinated contrast medium as a small renal portion laminated and stretched, located at the upper pole of the mass (Figure 2). The mass repressed the spleen and adrenal gland up without affecting their density. The stomach was distended and contained a water and food stasis. The contralateral kidney was in normal lumbar situation. Its size and density were normal. The liver was hypertrophic and multi nodular with nodules of variable size, hypodense with peripheral enhancement (Figure 2). The median vascular structures (aorta, inferior vena cava) were repressed and not invaded by this mass that discreetly crossed the median line. There was no thrombosis of the left renal vein but there was hypodensity in the inferior vena cava. The bony structures were undamaged and there was no disco-vertebral anomaly associated. CT concluded that it was left nephroblastoma with liver metastases. Chest X-ray showed no lung metastasis. The patient was transferred in pediatric surgery for better management. She had a laparotomy with left total nephrectomy. Pathological examination of the surgical specimen confirmed the diagnosis of Wilms tumor (Figures 3-5).

\section{Comments}

Nephroblastoma or Wilms tumor occurs in infancy. According to the literature 90\% of Wilms tumors occur be-

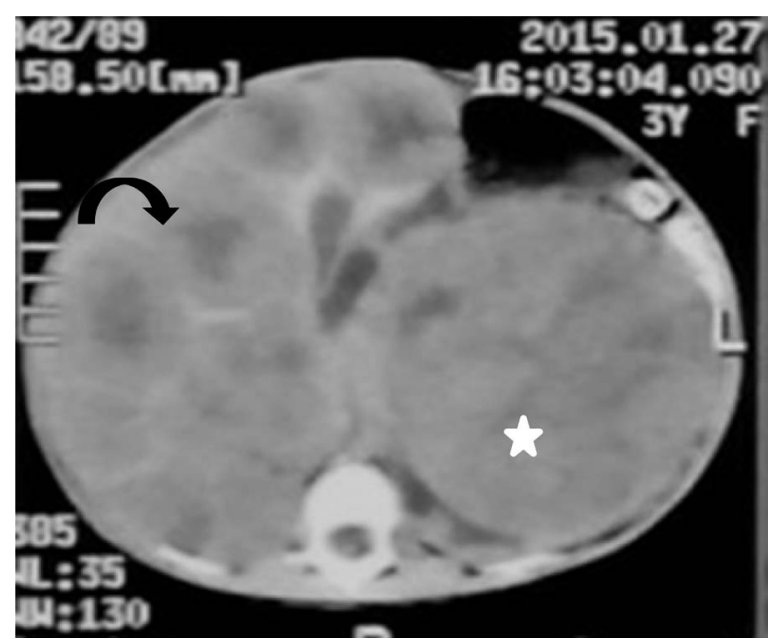

Figure 1. Abdominal CT in spontaneous contrast in a three-year-old girl with an abdominal-pelvic mass. Well limited rounded mass (star) without intratumor calcifications. Note liver nodules (arrow): Nephroblastoma stage IV. 


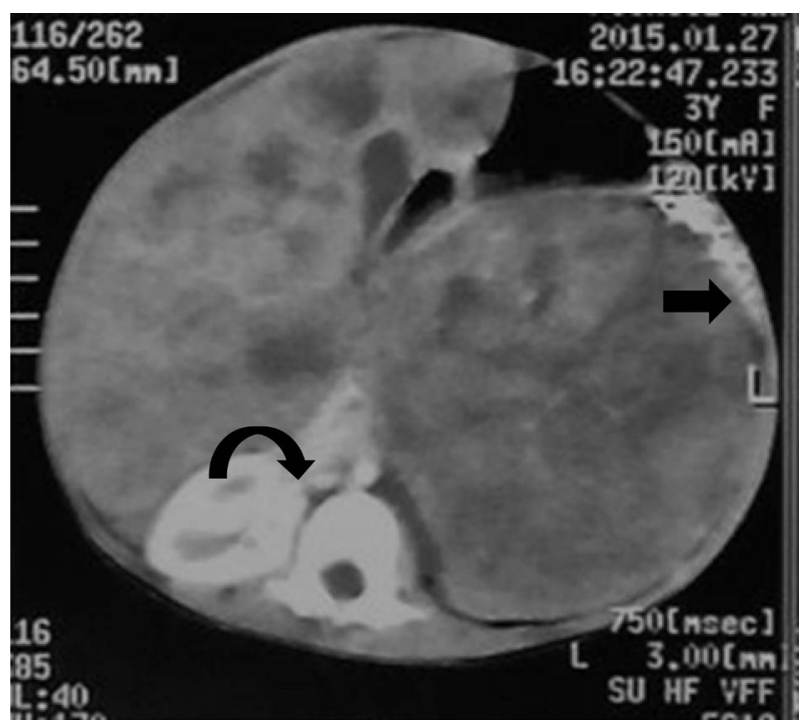

Figure 2. Same patient. CT after iodinated contrast medium injection. Moderate enhancement of the mass. The left kidney is rolled (Arrow). The median line is crossed by the tumor. The aorta and IVC are suppressed and not encompassed by the mass. Note hypodensity within the I VC (curved arrow).

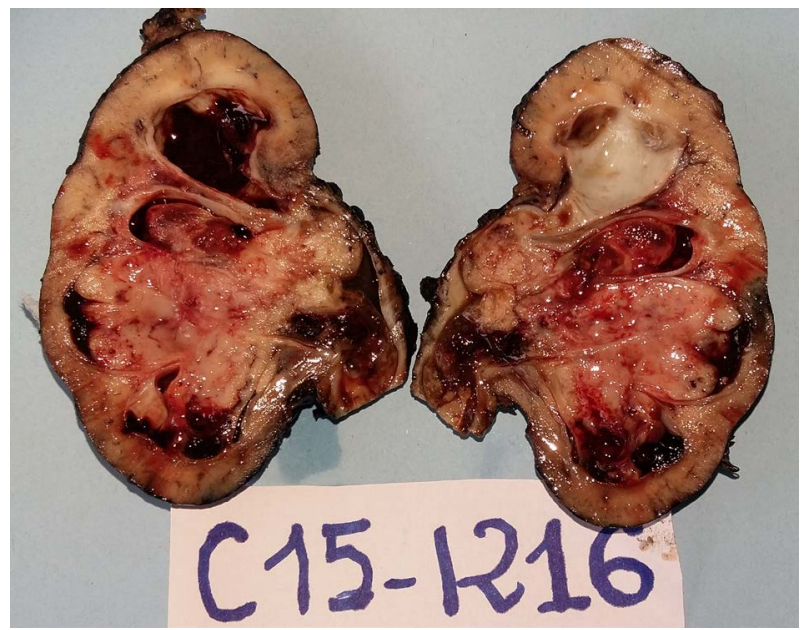

Figure 3. Left nephroureterectomy: cut surface showing a polychrome tumor, fleshy with cystic and hemorrhagic alterations. It measures $5 \mathrm{~cm}$ major axis and occupies the lower of the kidney starting cortex from the renal pelvis.

fore 07 years with a frequency peak at the age of 03 years [5]. Cases of Wilms tumor have been described in adolescents and adults [5]. In our case the patient's age is 03 years. The patient is female. This female predominance whatever light is found by Lubahn [6] in a series of 52 patients carriers of nephroblastoma (25 boys to 27 girls). Clinical signs of Wilms tumor in our case were dominated by a large abdominal mass. This is the most common cause of abdominal mass of the child [1] [2]. The volume of this mass is especially important in our case because of the delay in consultation in sub Saharan Africa [5]. Abdominal pain, preservation of the general condition and clinical anemia are usually described in this pathology. Other clinical signs such as hematuria and hypertension were not observed in our case. This could be explained by habits in sub Saharan Africa that involve the lack of systematic blood pressure measurement of children in consultation rooms (for lack of pediatric sphygmomanometer) and the absence of urinary dipsticks for systematic screening of microscopic hematuria. The plain film of the abdomen and abdominal ultrasound are the most available radiological examinations in the sub-Saharan African hospitals. If in presence of an abdominal mass, the plain film of the abdomen is not always required, ultrasound is the diagnostic method dedicated. It is well because of its safety especially in the pediatric field [7]. In our case the abdominal ultrasound was not very contributory. Although the left kidney was not visu- 


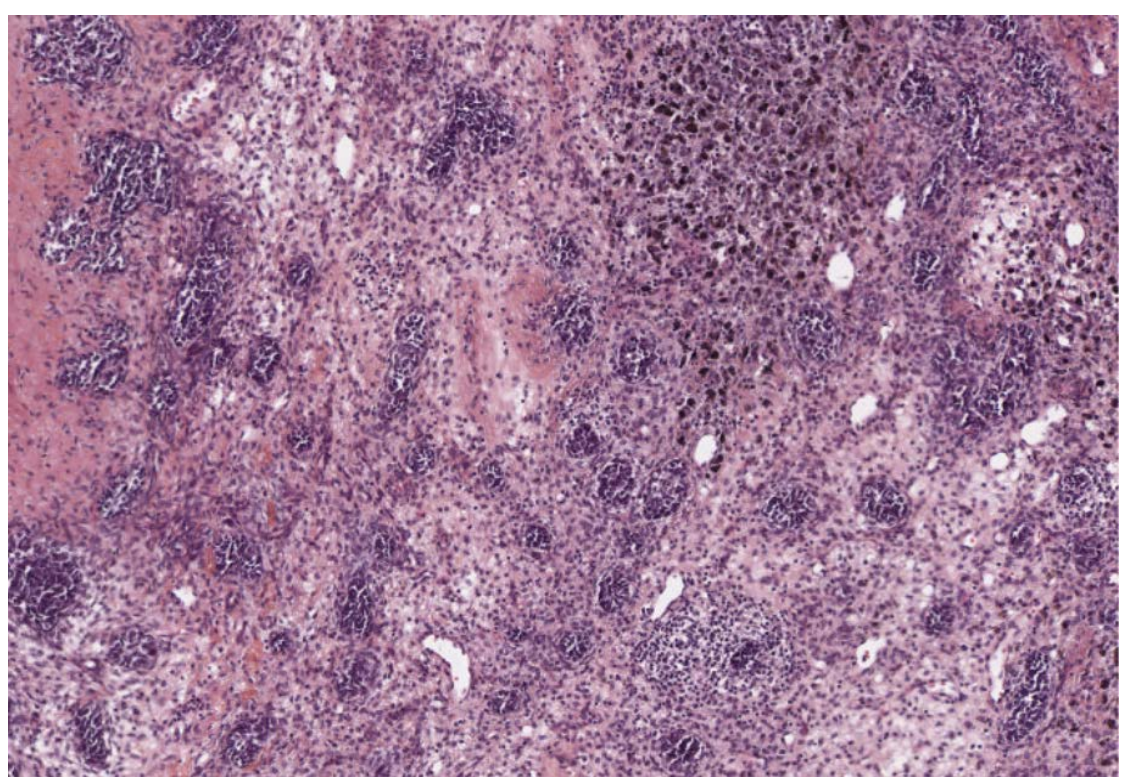

Figure 4. Hematoxylin-eosin $(\mathrm{HE}) \times 50$ : embryonal tumor proliferation made of cords, islets and pseudo rosettes.

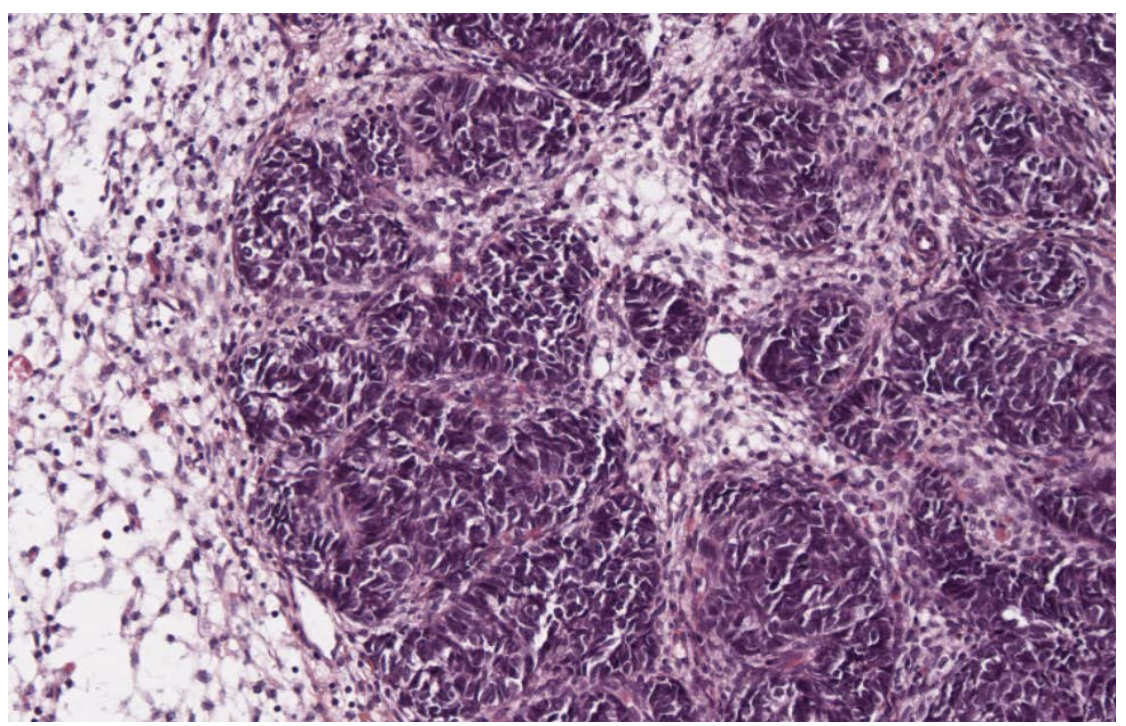

Figure 5. Hematoxylin-eosin $(\mathrm{HE}) \times 100$ : elongated or round cells tumor with a basophilic cytoplasm containing a hyperchromatic nucleus.

alized, the abdominal ultrasound did not make the organ diagnosis. Yet it has brought out the nature of the tissue mass. This allowed us to reduce the range of diagnostic hypotheses and suggest the indication of the other imaging methods. This range of diagnostic hypotheses in the presence of an abdominal mass of the child being among others according to Agboyega Olukayodede et al. [5] neuroblastoma, renal multi cystic disease, hydronephrosis and polycystic renal disease. In this perspective computed tomography (CT), scintigraphy, positron emission tomography and magnetic resonance imaging (MRI) are useful ways to make the diagnosis of Wilms tumor before histological confirmation [8]. This wide range of choices is conceivable only in developed countries. In our context, only CT is available. The radiation protection rules are therefore offset considering a high benefit/risk ratio. Several authors even in developed countries have performed a CT scan in the context of nephroblastoma [1]-[5], because the scanner has the advantage not only of bringing out the characteristics of the disease but also of making an accurate extension assessment and a clear pre-therapeutic staging [1]-[5]. In our case, the mass was large. It measured $132 \mathrm{~mm}$ in diameter. It was next to the left kidney that it rolled giving it the 
classic sign of the spur. It was of heterogeneous tissue density (55 UH). According to the literature, this heterogeneous pattern was related to necrosis and /or deposit of intratumor fat [9]. Conventionally there are internal calcifications which were not visualized in this case. These are coarse calcifications in opposition to neuroblastoma whose calcifications are fine and powdery. These calcifications were visualized in $17 \%$ of cases in the series of Agboyega et al. [5] and in 13\% of cases in the series of Geller et al [9]. The mass was very limited and did not invade the large vessels that it repressed. These intimate relationships between IVC and aorta show us that the mass is retro peritoneal. According to the literature nephroblastoma does not cross the median line unlike neuroblastoma. For Adegboyega et al. [5], the non-crossing of the median line is a dogma that we need to reconsider since in its series the majority of nephroblastomas crossed the median line probably because of their size and their discovery in the late stage. In our case, the median line is crossed by the mass. Signs usually present showing locoregional extension such as the invasion of the renal vein or lymph nodes that were not visualized. Paradoxically liver metastases were visualized, which allows classifying our nephroblastoma at stage IV [2] [10]. The paradox lies in the fact that metastases of Wilms tumor usually occur in the lungs and that it is neuroblastoma which has liver metastases.

\section{Conclusion}

Nephroblastoma or Wilms tumor, a common malignancy of children, is a typical example of malignancy whose diagnosis at the non-metastatic stages is hoped fervently. Indeed it is a malignant tumor whose cure on treatment is obtained $100 \%$ provided it is discovered early. Medical imaging, especially CT, is essential for positive diagnosis, pre therapeutic staging and monitoring of treatment. Hence the need to know the semiological elements of the positive and distinctive diagnosis of Wilms tumor compared to its main differential diagnosis is neuroblastoma. Some dogma such as the non crossing of the median line, the presence of coarse calcifications and lung metastases primarily pulmonary are to be reconsidered.

\section{Conflit D’Interets}

Aucun.

\section{References}

[1] Miele, V., Galluzzo, M., Bellussi, A. and Valenti, M. (1998) Spiral Computerized Tomography in the Study of Renal Neoplasms in Children. La Radiologia medica, 95, 486-492.

[2] Kim, S. and Chung, D.H. (2006) Pediatric Solid Malignancies: Neuroblastoma and Wilms Tumor. Surgical Clinics of North America, 86, 469-487. http://dx.doi.org/10.1016/j.suc.2005.12.008

[3] Visser, Y.T., Uys, R., van Zyl, A. and Stefan, D.C. (2014) Nephroblastoma: A 25-Year Review of a South African Unit. Journal of Medicine and Life, 7, 445-449.

[4] Lowe, R.E. and Cohen, M.D. (1984) Computed Tomographic Evaluation of Wilm's Tumour and Neuroblastoma. Radiographics, 4, 915-928. http://dx.doi.org/10.1148/radiographics.4.6.915

[5] Adegboyega Olukayode, A., Osuoji Richard, I., Akinola Rachael, A., Balogun Babajide, O., Faturoti Ireti, O. and Awosanya Gbolahan, O. (2014) Pattern of Computed Tomography Scan Findings in Children with Wilms' Tumor in a Tertiary Hospital in Lagos, Nigeria. Indian Journal of Medical and Paediatric Oncology, 35, 31-35. http://dx.doi.org/10.4103/0971-5851.133713

[6] Lubahn, J.D., Cost, N.G., Kwon, J., Powell, J.A., Yang, M., Granberg, C.F., Wickiser, J.E., Rakheja, D., Gargollo, P.C., Baker, L.A. and Margulis, V. (2012) Correlation between Preoperative Staging Computerized Tomography and Pathological Findings after Nodal Sampling in Children with Wilms Tumor. Journal of Urology, 188, 1500-1504. http://dx.doi.org/10.1016/j.juro.2012.02.020

[7] Cushing, B. and Slovis, T.L. (1992) Imaging of Wilms’ Tumor: What Is Important! Urologic Radiology, 14, $241-251$. http://dx.doi.org/10.1007/BF02926941

[8] Riccabona, M. (2003) Imaging of Renal Tumours in Infancy and Childhood. European Radiology, 13, 116-129. http://dx.doi.org/10.1007/s00330-003-2001-x

[9] Geller, E., Smergel, E.M. and Lowry, P.A. (1997) Renal Neoplasms of Childhood. Radiologic Clinics of North America, 35, 1391-1413.

[10] Abdelhalim, A., Helmy, T.E., Harraz, A.M., Abou-El-Ghar, M.E., Dawaba, M.E. and Hafez, A.T. (2014) Can Computerized Tomography Accurately Stage Childhood Renal Tumors? Journal of Urology, 192, 194-199. http://dx.doi.org/10.1016/j.juro.2014.01.096 